\title{
Frecuencia de enterobacterias nosocomiales productoras de $\beta$-lactamasas de espectro extendido, Cumaná, Venezuela
}

\section{Luzmila Sofía Albarado Ysasis' ${ }^{1}$ José García², Eliosmar Rodríguez², Carmen Carpio², Elsa Salazar³ , Evelin Margarita Flores Fernández', José Gregorio Betancourt' ${ }^{4}$, Yasmina Araque Calderón ${ }^{3}$, Militza Guzmán Lista ${ }^{3}$.}

1. Docente Categoría Agregado. 2. Licenciado en Bioanálisis

3. Docente Categoría Asociado. 4. Docente Categoría Asistente.

Universidad de Oriente, Núcleo de Sucre, Escuela de Ciencias, Departamento de Bioanálisis.

Correo electrónico: luzalv@hotmail.com

Recibido: 25-03-09 / Aceptado: 27-05-09

\section{Resumen}

El presente estudio tuvo como objetivo evaluar la frecuencia de enterobacterias nosocomiales productoras de $\beta$-lactamasas de espectro extendido en pacientes recluidos en el Servicio Autónomo Hospital Universitario "Antonio Patricio de Alcalá, Cumaná-Venezuela, durante el período septiembre-noviembre del 2005. La producción de $\beta$-lactamasas de espectro extendido y la susceptibilidad antimicrobiana in vitro, fue determinada por el método de sinergismo de doble disco y difusión en disco. Se obtuvieron 35 aislados bacterianos y el mayor número de Infecciones Intrahospitalarias se presentó en la unidad de cuidados intensivos y retén con I3/35 (37, I5\%) y 9/35 $(25,72 \%)$ casos, respectivamente.

El 5 I,42\% de cultivos positivos se presentó en muestras de secreciones. Klebsiella pneumoniae mostró una frecuencia de 20/35 (57, I 4\%). Sólo 27/35 aislados (77, I 4\%) produjeron $\beta$-lactamasas de espectro extendido y Klebsiella pneumoniae fue la especie más frecuente en la producción de éstas, con 14/35 (40,0\%). Las enterobacterias expresaron mayor porcentaje de resistencia a ceftazidima $(77,77 \%)$, cefotaxima $(70,37 \%)$ y cefepima (40,74\%). K. pneumoniae y E. coli, mostraron 59,25\% de resistencia a amoxicilina/ácido clavulánico. Todas presentaron $100 \%$ de sensibilidad a carbapenemas. Los largos períodos de hospitalización constituyen uno de los principales factores de riesgo para adquirir infecciones intrahospitalarias y la aparición de cepas productoras de $\beta$-lactamasas de espectro extendido resultan del mal uso de cefalosporinas de amplio espectro, lo cual plantea retos importantes por la necesidad de usar carbapenemas con el riesgo de ejercer una presión selectiva que cause, a futuro, su resistencia.

Palabras clave: $\beta$-lactamasas, cefalosporinas, enterobacterias, infecciones nosocomiales, susceptibilidad antimicrobiana.

\section{Abstract \\ Frequency of Nosocomial Enterobacterial $\beta$-lactamase-Producing of Extended Spectrum, Cumana, Venezuela}

This study aimed to evaluate the frequency of nosocomial enterobacterial $\beta$-lactamase producing extended spectrum in patients confined in the Hospital Universitario "Antonio Patricio de Alcalá, Cumana, Venezuela, 
during the period September-November 2005. The production of $\beta$-lactamases and extended spectrum in vitro antimicrobial susceptibility were determined by the method of double-disk synergy and disk diffusion. It took 35 bacterial isolates and the increased number of nosocomial infections occurred in the intensive care unit and neonatology with 13/35 (37.15\%) and 9 / 35 (25.72\%) cases respectively.

$51.42 \%$ of positive cultures occurred in samples of secretions. Klebsiella pneumoniae showed a frequency of 20/35 (57. I 4\%). Only 27/35 isolates (77. I 4\%) produced $\beta$-lactamases and extended spectrum Klebsiella pneumoniae was the most common species in the production of these, with I4/35 (40.0\%). Enterobacteriaceae expressed greater percentage of resistance to ceftazidime (77.77\%), cefotaxime (70.37\%) and cefepime (40.74\%). K. pneumoniae and E. coli, showed $59.25 \%$ resistance to amoxicillin / clavulanate. All showed I00\% sensitivity to carbapenems.

Long periods of hospitalization are major risk factors for acquiring nosocomial infections and the emergence of strains producing $\beta$-lactamases extended spectrum result from misuse of broad-spectrum cephalosporins, which poses significant challenges for the need to use carbapenems with the risk of exerting a selective pressure which in time will cause future resistance.

Keywords: antimicrobial susceptibility, $\beta$-lactams, cephalosporins, enterobacteria, nosocomial infectionsenterobacterial.

\section{Introducción}

La infección nosocomial o intrahospitalaria (IIH) representa uno de los mejores indicadores de calidad de atención debido a su frecuencia, la gravedad que conllevan, el aumento significativo de los costos que implica su ocurrencia y porque reflejan el resultado de acciones del equipo de salud (1). En el medio hospitalario, las áreas de cirugía y terapia intensiva, representan sitios propicios para el desarrollo de IIH por bacterias resistentes a agentes antimicrobianos (2). Entre los agentes bacterianos causantes se encuentran, principalmente, Acinetobacter baumannii, Klebsiella pneumoniae, Escherichia coli y Enterobacter spp. (3).

El amplio uso de los antibióticos, el incremento de la expectativa de vida, incluso en pacientes con enfermedades crónicas y la aparición de la unidad de cuidados intensivos (UCI), son factores que inciden directamente en la resistencia bacteriana a los antimicrobianos, y en el aumento de las IIH (4). Herrera y colaboradores señalan que este aspecto representa un problema de salud pública de carácter global (no existiendo país exento a este impacto) y una situación promovida, básicamente, por el uso indiscriminado de antibióticos en hospitales, clínicas, comunidad, agricultura y producción de alimentos (5).
Se conocen al menos siete mecanismos diferentes de resistencia a antimicrobianos, entre las enzimas más frecuentes que confieren resistencia están las $\beta$-lactamasas (6). La clasificación más utilizada para la nomenclatura de estas enzimas, ha sido la propuesta por Bush, Jacoby y Medeiros; el grupo 2b de esta clasificación, incluye las enzimas TEM-1, TEM-2 y SHV-1, conocidas como $\beta$-lactamasas de espectro extendido (BLEE), su presencia y permanencia en las bacterias es debido a la elevada presión selectiva ejercida por el uso irracional de la ampicilina, carbenicilina y CF1G (7-9). Adicional a las enzimas TEM y SHV, ha aparecido un nuevo grupo de BLEE, conocidas como cefotaximasas (CTX-M), las cuales presentan un grado de homología a nivel nucleotídico con TEM y SHV de $40 \%$ (10).

El Clinical and Laboratory Standards Institute (CLSI) sugiere evaluar agentes antimicrobianos como aztreonam, cefpodoxima, ceftazidima, cefatoxima y ceftriazona para realizar la detección de BLEE y confirmar la producción de estas enzimas en presencia de un agente inhibidor (11). El ácido clavulánico (AC) es un excelente inhibidor de las BLEE, usado al diseñar métodos para detectarlas al producir un fenómeno sinérgico entre $\mathrm{CF} 3 \mathrm{G}$, por ejemplo ceftazidima o cefotaxima y AC en ensayos por difusión en agar usando discos con estos compuestos (12). El uso 
indiscriminado de $\mathrm{CF} 3 \mathrm{G}$ en los centros hospitalarios, ha favorecido la aparición de importantes brotes nosocomiales causados por enterobaterias productoras de BLEE. La detección precoz de estas cepas y la aplicación de medidas de control adecuadas, favorecen la erradicación y vigilancia de estos brotes nosocomiales (13).

Se planteó como objetivo evaluar la frecuencia de enterobacterias nosocomiales productoras de BLEE en pacientes recluidos en el Servicio Autónomo Hospital Universitario "Antonio Patricio de Alcalá (SAHUAPA), en el período septiembre- noviembre de 2005.

\section{Materiales y métodos}

Durante el período septiembre-noviembre de 2005, se recolectaron 35 cepas bacterianas de la familia Enterobacteriaceae, identificadas en el laboratorio de bacteriología del SAHUAPA, aisladas de pacientes con $\mathrm{IIH}$ recluidos en las áreas de UCI, retén, medicina y pediatría del centro hospitalario, respetando para la selección de las muestras, los criterios establecidos por el Center for Diseases Control (CDC) (14). Las cepas bacterianas fueron inoculadas en medio de conservación, agar tripticasa de soya (Himedia) con 50\% de glicerol, y transportadas al laboratorio de Microbiología General y Bacteriología Clínica del Departamento de Bioanálisis de la Universidad de Oriente-Núcleo Sucre, Venezuela, para su reidentificación. Por tratarse de un trabajo de investigación, incluyó un estudio bacteriológico completo: siembra en medio básico, selectivo diferencial, evaluación microscópica e identificación bioquímica por métodos convencionales.

El estudio bacteriológico se realizó siguiendo lineamientos de Koneman y su equipo (15) para la identificación de enterobacterias. A partir del medio de conservación, las cepas bacterianas se inocularon en caldo infusión cerebrocorazón, BHI (Himedia), y se incubaron por 24 horas a $37^{\circ} \mathrm{C}$ en aerobiosis. Luego, se sembraron en agar tripticasa de soya, ATS (Himedia) y agar MacConkey, AMC (Himedia), incubándose en iguales condiciones (16). La evaluación macroscópica de las colonias se realizó por observación de sus características morfológicas y cambios producidos en el AMC (15). El estudio microscópico, se realizó preparando un extendido a partir del cultivo en ATS, luego, se procedió a colorear con el método de Gram (17), para establecer morfología y tinción bacteriana. La caracterización bioquímica de las cepas se realizó según protocolos de identificación convencional para bacterias Gram negativas fermentadoras y no fermentadoras de lactosa, propuestos por Koneman y colaboradores (15) y Mac Faddin (16).

La susceptibilidad antimicrobiana fue realizada mediante el método de difusión del disco (18), siguiendo los lineamientos propuestos para enterobacterias por el CLSI (11), empleando discos de antibióticos de la casa comercial OXOID: amoxacilina-ácido clavulánico 2:1 $(20 \mu \mathrm{g} / 10 \mu \mathrm{g})$, cefotaxima $(30 \mu \mathrm{g})$, ceftazidima $(30 \mu \mathrm{g})$, cefepima $(30 \mu \mathrm{g})$, imipenem $(10 \mu \mathrm{g})$, meropenem $(10 \mu \mathrm{g})$. Los resultados se reportaron según recomendaciones del CLSI (11) en las categorías de interpretación de acuerdo a los datos obtenidos in vitro (19).

La producción de BLEE se determinó por la técnica de sinergismo de doble disco propuesta por Jarlier y colaboradores (20) y siguiendo los lineamientos establecidos por CLSI (11), M100-S15 (M2), aplicándose a las cepas recolectadas de Klebsiella spp, E.coli y también a Enterobacter spp. Para ello, se colocó en el centro de una placa de agar Mueller Hinton (DIFCO) un disco de amoxacilina/ácido clavulánico 2:1 $(20 \mu \mathrm{g} / 10 \mu \mathrm{g})$ y los discos de ceftazidima y ceftriazona a una distancia lineal de $20 \mathrm{~mm}$ del disco central en un ángulo de $90^{\circ}$, a $20 \mathrm{~mm}$ del mismo se ubicaron los discos de aztreonam y cefotaxima. La formación de un óvalo en la zona de conversión del halo de inhibición de los agentes antimicrobianos empleados y el disco central de amoxacilina-ácido clavulánico y la sensibilidad ante cefoxitina, es indicativo, desde el punto de vista fenotípico, de la presencia de BLEE. La calidad de los discos de antibióticos, así como la producción de $\beta$-lactamasas, fue controlada empleando las cepas de $E$. coli ATCC 25922, especie BLEE negativa, y $K$. pneumoniae ATCC 700603, productora de BLEE (11).

Para establecer la frecuencia de bacterias productoras de BLEE, se empleó un análisis porcentual. Asimismo, se aplicó un análisis de múltiples variables para correlacionar las enterobacterias nosocomiales identificadas, producción de BLEE, tiempo de estadía en el centro hospitalario, tipo de muestra analizada y área de hospitalización, a un nivel de confiabilidad de $95 \%$ (21). 
Tabla 1. Distribución porcentual del número de casos de infecciones nosocomiales causadas por enterobacterias, según las áreas de hospitalización.

\begin{tabular}{lcc} 
Área de hospitalización & $\mathrm{N}^{\circ}$ de casos & $\%$ \\
Unidad de cuidados intensivos & 13 & 37,15 \\
Retén & 9 & 25,72 \\
Medicina C & 6 & 17,14 \\
Medicina A & 3 & 8,57 \\
Medicina B & 2 & 5,71 \\
Pediatría A & 2 & 5,71 \\
Total & 35 & 100,00 \\
\hline
\end{tabular}

Servicio Autónomo Hospital Universitario "Antonio Patricio de Alcalá", Cumaná, Venezuela. Septiembrenoviembre de 2005.

Tabla 2. Frecuencia de cultivos positivos para enterobacterias nosocomiales, según el tipo de muestra clínica.

$\begin{array}{lcc}\text { Muestra } & \text { Cultivos positivos } & \% \\ \text { Secreciones } & 18 & 51,42 \\ \text { Catéter } & 8 & 22,86 \\ \text { Sangre } & 5 & 14,29 \\ \text { Heces } & 2 & 5,71 \\ \text { Líquido cefalorraquídeo } & 1 & 2,86 \\ \text { Orina } & 1 & 2,86 \\ \text { Total } & 35 & 100,00\end{array}$

Servicio Autónomo Hospital Universitario "Antonio Patricio de Alcalá", Cumaná, Venezuela. Septiembrenoviembre de 2005.

\section{Resultados}

Se obtuvieron 35 aislados bacterianos de pacientes con IIH; el estudio indicó que el mayor número de casos se presentó en UCI y retén con $13(37,15 \%)$ y 9 (25,72\%) casos, respectivamente; asimismo, en las áreas de Medicina $\mathrm{B}$ y Pediatría A se obtuvo menor cantidad, cada uno con 2 casos (5,71\%), Tabla 1 . Según el tipo de muestra clínica, $51,42 \%$ (18) de los cultivos bacteriológicos positivos para enterobacterias nosocomiales, se presentó en muestras de secreciones, seguidos de $22,86 \%$ (8) en muestras de catéter. En la Tabla 2 se relacionan las frecuencias para enterobacterias nosocomiales. En la Tabla 3 se muestran el número de cepas y el porcentaje que representan de los aislados clínicos.

La detección fenotípica de BLEE indicó que de 35 aislados bacterianos, 27 fueron productores de estas enzimas, lo que representa $77,14 \%$, la producción en $K$. pneumoniae fue $14 / 20$, correspondiéndose con $40,00 \%$, seguido de E. aerogenes, donde 5 aislados resultaron productores con 14,29\%, Tabla 4 . 
Tabla 3. Frecuencia de enterobacterias nosocomiales.

\begin{tabular}{lcc} 
Especie bacteriana & $\mathrm{N}^{\circ}$ de cepas & $\%$ \\
Klebsiella pneumoniae & 20 & 57,14 \\
Enterobacter aerogenes & 6 & 17,14 \\
Escherichia coli & 4 & 11,43 \\
Enterobacter spp. & 3 & 8,57 \\
Klebsiella oxytoca & 2 & 5,71 \\
Total & 35 & 100,00 \\
\hline
\end{tabular}

Servicio Autónomo Hospital Universitario "Antonio Patricio de Alcalá", Cumaná, Venezuela. Septiembrenoviembre de 2005.

Tabla 4. Frecuencia de enterobacterias nosocomiales productoras de $\beta$-lactamasas de espectro expandido.

\begin{tabular}{lcrcr} 
Especie bacteriana & BLEE + & $\%$ & BLEE - & $\%$ \\
\hline Klebsiella pneumoniae & 14 & 40,00 & 6 & 17,14 \\
Enterobacter aerogenes & 5 & 14,29 & 1 & 2,86 \\
Escherichia coli & 3 & 8,57 & 1 & 2,86 \\
Enterobacter spp. & 3 & 8,57 & 0 & 0 \\
Klebsiella oxytoca & 2 & 5,71 & 0 & 0 \\
Total & 27 & 77,14 & 8 & 22,86 \\
\hline
\end{tabular}

Servicio Autónomo Hospital Universitario "Antonio Patricio de Alcalá", Cumaná, Venezuela. Septiembre-noviembre de 2005. BLEE: $\beta$-lactamasas de espectro expandido.

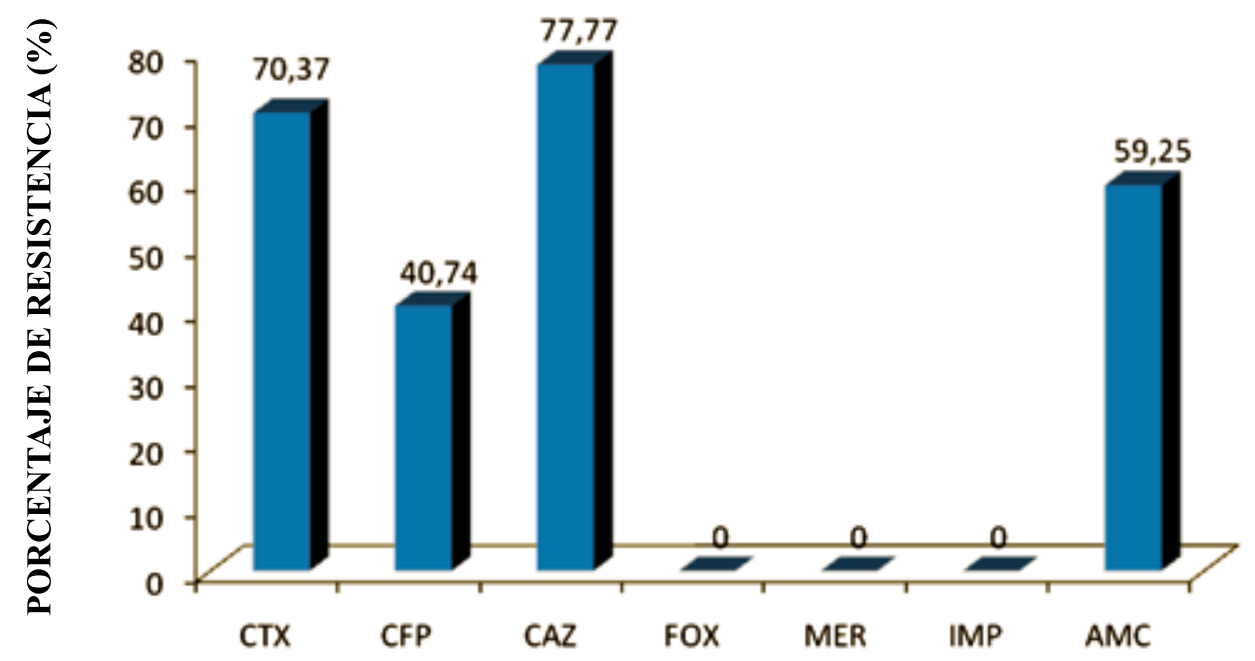

\section{ANTIBIÓTICOS BETALACTÁMICOS}

Figura 1. Susceptibilidad in vitro a los antibacterianos $\beta$-lactámicos. CTX: cefotaxima, CFP: cefepima; CAZ: ceftazidima, FOX: cefoxitina (excepto Enterobacter spp.), IPM: imipenem, MER: meropenem, AMC: amoxicilina/ácido clavulánico (sólo para K. pneumoniae y E. coli). 
Las pruebas de susceptibilidad in vitro a los antibacterianos $\beta$-lactámicos muestran que las enterobacterias nosocomiales expresaron mayor porcentaje de resistencia a ceftazidima con $77,77 \%$ y a cefotaxima con $70,37 \%$. En la Figura 1 se muestra el porcentaje de susceptibilidad a los antibióticos $\beta$-lactámicos.

\section{Discusión}

Los factores clínicos epidemiológicos evaluados, los largos períodos de hospitalización y la administración de antimicrobianos representan factores que condicionan el establecimiento del proceso infeccioso en los pacientes. Según Martín y colaboradores (22) la frecuencia de IIH en pacientes de UCI está asociada al alto consumo de antimicrobianos, el cual es aproximadamente diez veces mayor en la UCI que en el resto de las áreas hospitalarias y a las características de los pacientes allí hospitalizados, su prolongada estadía y características medio ambientales propias, que contribuyen con una ecología favorable a los microorganismos. Alpuche y Daza (23) en un estudio sobre frecuencia de bacterias nosocomiales resistentes a cefalosporinas de espectro extendido, mostró que entre 35 y 40\% de las IIH procedían de UCI. Pérez publicó que las UCI representan, en muchos casos, el recurso más idóneo para salvar la vida de los pacientes en estado crítico, sin importar la causa de su ingreso, pero esa esperanza de vida tiende a complicarse dado que las UCI están propensas a albergar microorganismos causantes de infección nosocomial (24).

Este estudio mostró un $51,42 \%$ de cultivos positivos para enterobacterias nosocomiales a partir de secreciones. Resultados relacionados fueron publicados por Poveda y su equipo (25), quienes en un estudio sobre infecciones nosocomiales en terapia intensiva, mostraron un $79 \%$ de cultivos positivos en secreciones. $K$. pneumoniae fue la especie bacteriana mayormente aislada $(57,14 \%)$. Estos resultados guardan relación con un trabajo realizado por Poveda y colaboradores (25), donde Klebsiella pneumoniae fue el principal agente causal de IIH (33,30\%). Cornejo y colaboradores (28), en México, publicaron una investigación sobre patrones de resistencia bacteriana en urocultivos, analizando por separado cepas nosocomiales y de la comunidad, E. coli ocupó el primer lugar en muestras nosocomiales con $41,3 \%$.
En este estudio se obtuvo un $77,14 \%$ de enterobacterias nosocomiales productoras de BLEE; Klebsiella pneumoniae resultó el principal productor de las enzimas. El mayor porcentaje de aislados bacterianos resistentes a antimicrobianos $\beta$-lactámicos, se obtuvo para ceftazidima $(77,37 \%)$, cefotaxima $(70,37 \%)$ y cefepima $(40,74 \%)$, siendo totalmente sensibles a las carbapenemas ensayadas. Perozo-Mena y colaboradore (29) estudiando BLEE en cepas de E. coli y $K$. pneumoniae aisladas en pacientes de UCI en un hospital de Maracaibo-Venezuela, obtuvieron que $16 / 46(39,02 \%)$ cepas de E. coli y $31 / 59$ (52,54\%) de $K$. pneumoniae resultaron positivas a la prueba en un total de 100 cepas evaluadas.

Araya y su equipo (30) en una publicación sobre infecciones por bacterias Gram negativas en pacientes internados en el Hospital San Juan de Dios de Costa Rica, indicaron como principales agentes bacterianos a $E$. coli y $K$. pneumoniae, destacando entre los $\beta$-lactámicos, una alta resistencia a piperacilina-tazobactam $(78 \%)$ y cefepima (100\%), y sólo el imipenem fue activo (100\%) en los aislamientos. Por otro lado, Torres y colaboradores (31) en un estudio sobre BLEE en enterobacterias aisladas en Centros de Salud de Caracas, Venezuela, reportaron que de $91,10 \%$ de las cepas productoras de BLEE, 5,80\% mostraron resistencia a cefepima, destacando que debido a que las BLEE sufren del efecto inóculo, en aquellas infecciones con inóculos bacterianos elevados, cefepima puede ser hidrolizada con mayor afinidad y velocidad, conduciendo a un fracaso terapéutico.

Enterobacter spp., al igual que Citrobacter freundii, Morganella morganii, Providencia stuartii y Providencia rettgeri, producen $\beta$-lactamasas cromosómicas inducibles denominadas AmpC, no inhibidas por ácido clavulánico y otros inhibidores de $\beta$-lactamasas (32). Las bacterias productoras de estas enzimas son resistentes a las aminopenicilinas y a las cefalosporinas de primera, segunda y tercera generación. La resistencia a cefoxitina varía entre las distintas especies y depende del nivel de expresión de la enzima cromosómica. En algunos casos la hiperproducción de estas enzimas puede otorgar resistencia a dichas cefalosporinas (33).

Cortés y colaboradores defienden que el factor de riesgo que más se asocia con la producción de BLEE es el tiempo prolongado de hospitalización, bien sea en un 
servicio médico quirúrgico o en la UCI, o el antecedente de hospitalización y el uso previo de múltiples esquemas de antibióticos, particularmente, CF3G, trimetoprim sulfametoxazol y aminoglucósidos, también señalan, que la presión selectiva que ha llevado a la evolución de las BLEE ha sido usualmente atribuida al uso amplio e indiscriminado de las CF3G (34). Según Torres y su equipo, la presencia de plásmidos de alto peso molecular en las bacterias sugiere que la presión ejercida por el uso de antibióticos en áreas como UCI, ha favorecido la diseminación y prevalencia no sólo de plásmidos transferibles, sino de otros elementos genéticos como transposones e integrones que codifican altos niveles de resistencia (31). Esto pudiera estar diseminándose fácilmente entre los aislados bacterianos que producen IIH en las distintas áreas del centro asistencial, probablemente, debido al excesivo uso de $\beta$-lactámicos de amplio espectro.

Los largos períodos de hospitalización constituyen uno de los principales factores de riesgo para adquirir $\mathrm{IIH}$, el uso de antibióticos ß-lactámicos es muy utilizado para su tratamiento, por su baja toxicidad y amplio espectro; sin embargo, la aparición de cepas productoras de BLEE, resultan del mal uso de cefalosporinas de amplio espectro que ejercen una presión selectiva y favorecen la aparición de mecanismos de resistencia, lo cual plantea retos importantes desde el punto de vista terapéutico, ya que la única opción sería el uso de carbapenemas, con el riesgo de ejercer una presión selectiva que desarrolle su resistencia.

\section{Agradecimientos}

Los autores desean expresar su agradecimiento a aquellas personas que participaron en este trabajo y al consejo de investigación de la Universidad de Oriente, Núcleo de Sucre, por el apoyo financiero en el Proyecto identificado con el código No CI-2-040102-1409/08. NOV

\section{Referencias}

1. Guinan JL, McGuckin M, Shubin A, Tighe J. A descriptive review of malpractice claims for health care-adcquired infections in Philadelphia. Am J Infect Control. 2005;33:310-312.

2. Casellas J. Resistencia bacteriana por producción de $\beta$-lactamasas de espectro extendido: La perspectiva global y latinoamericana en el escenario hospitalario. Reunión de consenso-La aparición de microorganismos productores de ESBL en América Latina: Recomendaciones para su control y tratamiento (Sao Pablo, Brasil). Infec Dis Clin Pract. 2001;12-16.
3. Díaz R, Solórzano F, Padilla G, Miranda M, González R, Trejo J. Infecciones nosocomiales. Experiencia en un hospital pediátrico de tercer nivel. Salud Púb Mex. 2001;41:12-17.

4. Owens R y Rice L. Hospital-based strategies for combating resistance. Clin Infect Dis. 2006;42:S173-181.

5. Herrera K, Espinoza M, Mejía Y, Zambrano L, Silva E, Rojas J, Gadea W, Cavaría S, Hernández M, Ramírez M, Membreño J, Lara M, Saenz J, Valle S, Torrez A, Carera E, Cáceres M. Resistencia antimicrobiana en Hospitales nor-occidentales de Nicaragua. Universitas. 2007;1:27-32.

6. Goodman R. y Gilman J. Las bases farmacológicas de la Terapéutica. Novena edición. Editorial Médica Panamericana. Buenos Aires. 1999.

7. Ambler R. The structure of beta-lactamases. Philos Trans R Soc Lond B Biol Sci. 1980;289:321-331

8. Bush K, Jacoby G, Medeiros A. A functional classification scheme for B-lactamases and its correlation with molecular structure. Antimicrob Agents Chemother. 1995;39:1211-1233.

9. Rice L. Evolution and Clinical Importante of Extended-Espectrum $\beta$-Lactamases. Chest. 2001;119:391-396.

10. Bradford P. Extended-spectrum $\beta$-lactamases in the 21 st century: characterization, epidemiology, and detection of this important resistance threat. Clin Microbiol Rev. 2001;14:933-935.

11. Clinical and Laboratory Standards Institute (CLSI). Performance Standards for Antimicrobial Susceptibility Testing; Fifteenth Informational Supplement. 2007; 25:178.

12. Blásquez J, Baquero M, Canton R, Alos I, Baquero F. Characterization of a new TEM-type $\beta$-lactamase resistant to clavulanate, sulbactam and tazobactam in a clinical isolate of Escherichia coli. Antimicrob Agents Chemother. 1993;37:2059-2063.

13. Murria P, Baron E, Jorgensen J. Manual of Clinical Microbiology. Octava edición. ASM USA. 2003.

14. Horan TC, Andrus M, Dudeck MA. CDC/NHSN surveillance definition of health care-associated infection and criteria for specific types of infections in the acute care setting. Am J Infect Control 2008;36:309-332.

15. Koneman E, Allen S, Janda W, Schreckenberger P, Washington, W. Diagnóstico Microbiológico. Quinta edición. Editorial Panamericana. 2002

16. Mac Faddin J. Pruebas bioquímicas para la identificación de bacterias de importancia clínica. Quinta edición. Editorial Panamericana. Buenos Aires. 2003.

17. Huccker G. y Coon H. Methods of Gram Staining. Technical Bulletin New Cork State Agricultura Experimentation. 1923;93:1-37.

18. Bauer A, Kirby W, Sherris J, Turck M. Antibiotic susceptibility testing by a standardized single disk method. Am J Clin Pathol. 1966;45:493-496.

19. Crespo M. La lectura interpretativa del antibiograma: Una herramienta para predecir la resistencia bacteriana en el laboratorio de microbiología de rutina. Colomb Med. 2002;33:179-193.

20. Jarlier V, Nicolas M, Fournier G, Philippon A. Extended broadspectrum b-lactamases conferring resistance to newer $\beta$-lactam agents in Enterobacteriaceae: hospital prevalence and susceptibility patterns. Rev Infect Dis. 1989;10:867-878.

21. Dawsen S. y Robert G. Bioestadística Médica. Editorial el Manual Moderno S.A. México DF. 1997.

22. Martín G, Carmona O, Guzmán M. Infección nosocomial II: Resistencia a ß-lactámicos y aminoglucósidos en Pseudomonas aeruginosa en centros médicos de Venezuela durante el año 2000. Rev Soc Ven Microbiol. 2003;23: 183-189.

23. Alpuche M. y Daza C. Infecciones nosocomiales por bacterias Gram negativas resistentes a cefalosporinas de espectro extendido: asociación de dos peligrosos enemigos. Enf Infec y Microbiol. 2002;22:192-199. 
24. Pérez C. Antimicrobianos en Unidades de Cuidados Intensivos: Uso empírico. Rev chil Infectol. 2003;20:70-73.

25. Poveda L, Villamizar D, Sánchez F, Otta A, Guevara C, Jiménez $\mathrm{M}$, Besso J. Infecciones nosocomiales en terapia intensiva. Antib Infect. 2005;2:40.

26. Muzachiodi M, Ferrero S. Incidencia de enterobacterias productoras de betalactamasas de espectro extendido en el Hospital Escuela José F. de San Martín. Facultad de Cs. Exactas Nat. y Agrim. Dpto. de Bioquímica. Argentina. 2005.

27. Martínez P, Mercado M, Máttar S. Determinación de $\beta$-lactamasas de espectro extendido en gérmenes nosocomiales del Hospital San Jerónimo, Montería. Colomb Med. 2003;34:130-139.

28. Cornejo P, Velásquez C, Sandoval S, Gordillo P, Volkow P. Patrones de resistencia bacteriana en urocultivos en un hospital oncológico. Salud Púb Méx. 2007:49:330-336.

29. Perozo-Mena A, Castellano-González M, Ginestre-Pérez M, Harris B. Caracterización Molecular y Detección de Betalactamasas de Espectro Extendido en Cepas de E. coli y K. pneumoniae Aisladas en las Unidades de Cuidados Intensivos de un Hospital Universitario. Kasmera. 2007;35:91-106.
30. Araya C, Boza R, Arguedas L, et al. Infecciones nosocomiales por bacterias productoras de betalactamasas de espectro ampliado: prevalencia, factores de riesgo y análisis molecular. Acta Méd Costarric. 2007;49:90-96.

31. Torres L, Gagliotta V, Torres O, Benítez M, Domínguez M, Pedroza R. $\beta$ Lactamasas de Espectro Expandido en Enterobacterias aisladas en Centros de Salud de Caracas. Rev Soc Ven Microbiol. 2006;26:190-205.

32. Livermore D. $\beta$-lactamases in laboratory and clinical resistance. Clin Microbiol Rev. 2000;8:557-584.

33. Famiglietti $\mathrm{A}$, Quinteros $\mathrm{M}$, Vázquez $\mathrm{M}$, Marín $\mathrm{M}$, Incola $\mathrm{F}$, Radice M. Consenso sobre las pruebas de sensibilidad a los antimicrobianos en Enterobacteriaceae. Rev Argent Microbiol. 2005;37:57-66.

34. Cortés J, Urdaneta A, Potdevin G, Cuervo S, Bermúdez D, Molina C, Arroyo, P. Impacto de las betalactamasas de espectro extendido en pacientes con cáncer. Rev Colomb Cancerol. 2006;10:183-196. 